\title{
Man-made Object Detection Based on Texture Visual Perception
}

\author{
Fei Cai, Honghui Chen, Jianwei Ma \\ Department of Science and Technology on Information Systems Engineering National University of Defense \\ Technology ChangSha, HuNan Province, China
}

\begin{abstract}
Based on human visual attention mechanism and texture visual perception, this paper proposes a method for man-made object detection by extracting texture and geometry structure features. Followed by clustering the texture feature, geometry structure feature is obtained to realize final detection. Then a man-made object detection scheme is designed, by which typical man-made objects in complex natural background, including airplanes, tanks and vehicles can be detected. The experiments sustain that the proposed method is effective and rational.
\end{abstract}

Index Terms: Man-made object detection; image interpretation; feature extraction; clustering

(C) 2012 Published by MECS Publisher. Selection and/or peer review under responsibility of the Research Association of Modern Education and Computer Science.

\section{Introduction}

As a key technology of image processing, man-made object detection is the basis for target tracking and recognition, widely applied in intelligent transportation[1], urban planning[2], video surveillance[3] and military target detection[4]. It can improve the efficiency of image interpretation, and has great significance for intelligence obtaining and situation grasping of a war.

Several man-made object detection methods, based on fractal feature, probability models, level set and clustering, are generalized in [5]. The main difficulty lies in obtaining significant differences between man-made objects and background to achieve effective segmentation, Concluding from human visual perception, a new man-made object detection approach, involving texture clustering and geometric structure feature extracting, is proposed, Theoretical analysis and experimental comparison verifies the rationality of proposed approach.

\section{Background}

In image processing field, man-made object detection is classified into image segmentation. An image

* Corresponding author.

E-mail address: fei525lin@163.com,chh0808, majianwei.nudt@google.com 
$G(x, y) \quad$ can be described as $(1)$
$G(x, y)=B(x, y)+\sum_{i=1}^{N} O_{i}(x, y)+N(x, y)$

where $B(x, y)$ represents background, $O_{j}(x, y)$ means man-made object and $N(x, y)$ is noise, then the main process can be generalized as Fig.1. Combining with practical application, this paper proposes the following three assumptions: (1) Visual discontinuity between objects and background does exist; (2) The geometric structure features of man-made object are more prominent than background; (3) The frequency of occurrence of man-made objects is low compared to background.

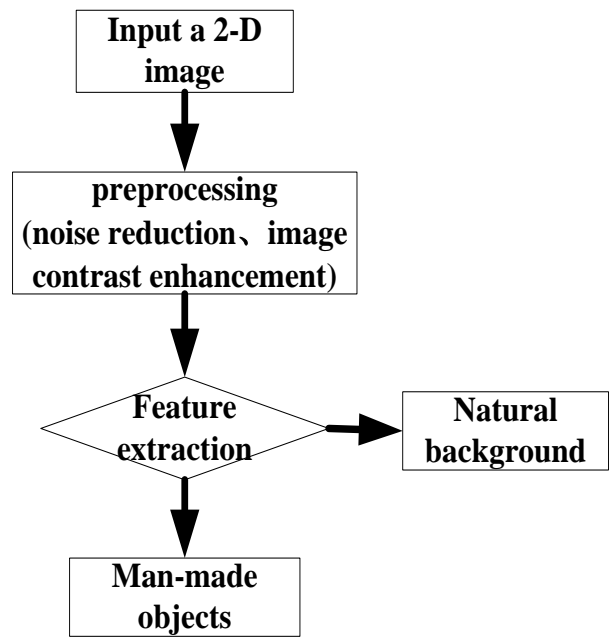

Fig. 1 man- made object detection flow chart.

\section{Proposed Algorithm}

The framework of proposed algorithm for man-made object detection is shown in Fig.2.

\section{A. Preprocessing}

Salt and pepper noise in acquiring images and Gaussian noise caused principally by image transmission are taken into account. Furthermore, we enhance the contrast by mathematic morphology after measuring it by DCT coefficients. Owing that human visual detection depends on the ratio between high-frequency and low-frequency content [7], the contrast can be measured as the ratio of high-frequency and low-frequency content in the bands of the DCT matrix $[6,7]$.

\section{B. objects primary location}

The paper compares the results between histogram-based and Tamura-based texture clustering. Ultimately, contrast based on Tamura is chosen and FCM is employed as the clustering algorithm. 


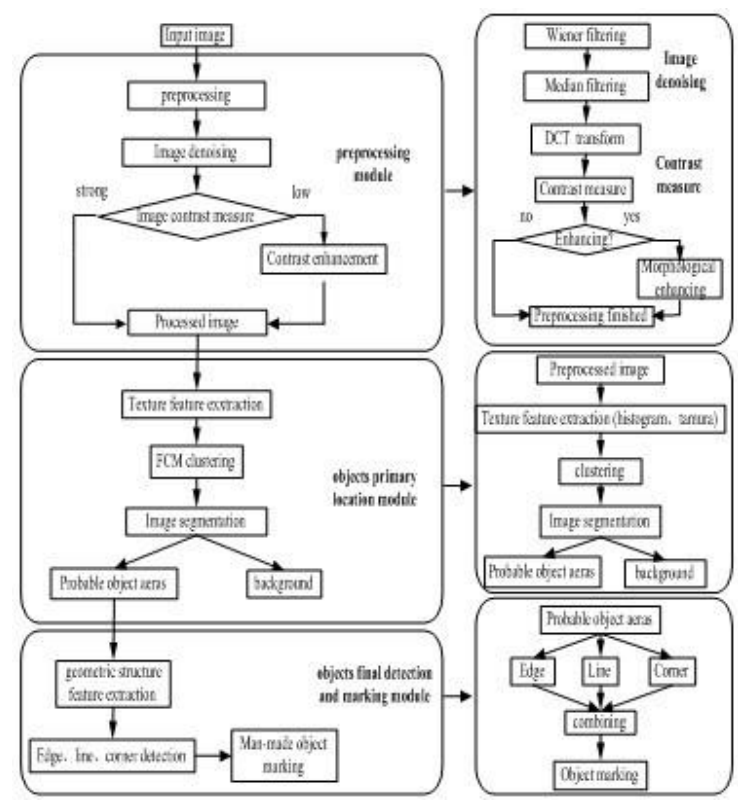

Fig. 2 experimental framework

\section{C. objects primary location}

The paper compares the results between histogram-based and Tamura-based texture clustering. Ultimately, contrast based on Tamura is chosen and FCM is employed as the clustering algorithm.

One approach for describing texture is to use statistical moments of histogram. Let $z$ be a random variable, the nth moment of $z$ is

$$
\mu_{n}=\sum_{i=0}^{L-1}\left(z_{i}-m\right)^{n} p\left(z_{i}\right)
$$

where $\mathrm{L}$ is the number of gray levels, $\mathrm{m}$ is the mean value of $z$

$$
m=\sum_{i=0}^{L-1} z_{i} p\left(z_{i}\right)
$$

Standard deviation $\sigma$ measures the contrast.

$$
\sigma=\sqrt{\mu_{2}(z)}=\sqrt{\sigma^{2}}
$$

Tamura[8] proposed other statistics to describe texture based on the psychological research on visual perception. Contrast also can be derived as

$$
F_{\text {contrastness }}=\frac{\sigma}{\alpha_{4}^{1 / 4}}
$$

where $\alpha_{4}=\mu_{4} / \sigma^{4}, \mu_{4}$ is the 4 th moment and $\sigma^{2}$ is the variance. 


\section{D. objects final detection and marking}

Man-made objects can be marked after lines and corners detection on the basis of primary location. Susan operator[9] is employed to detect corners and edges are detected by Tupin approach[10,11]. Fig.3 shows the partial experimental results.

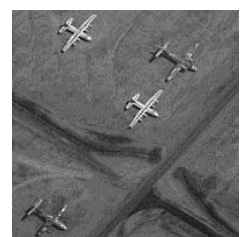

(a) Original image

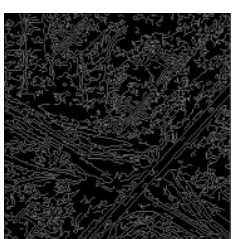

(b) Canny

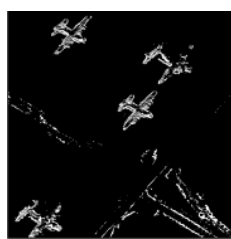

(c) Tupin approach

Fig. 3 Distinct edge detection results

\section{Experiment Results and Discussion}

The experiment is carried out as follows: (1)Input a gray image, enhance the contrast after denoising with Weiner and median filter, (2)Extract texture feature;(3)Cluster texture feature to realize image segmentation by FCM;(4)Extract the geometric structure feature, to mark the objects.

TABLE I. THE RESULTS OF PREPROCESSING

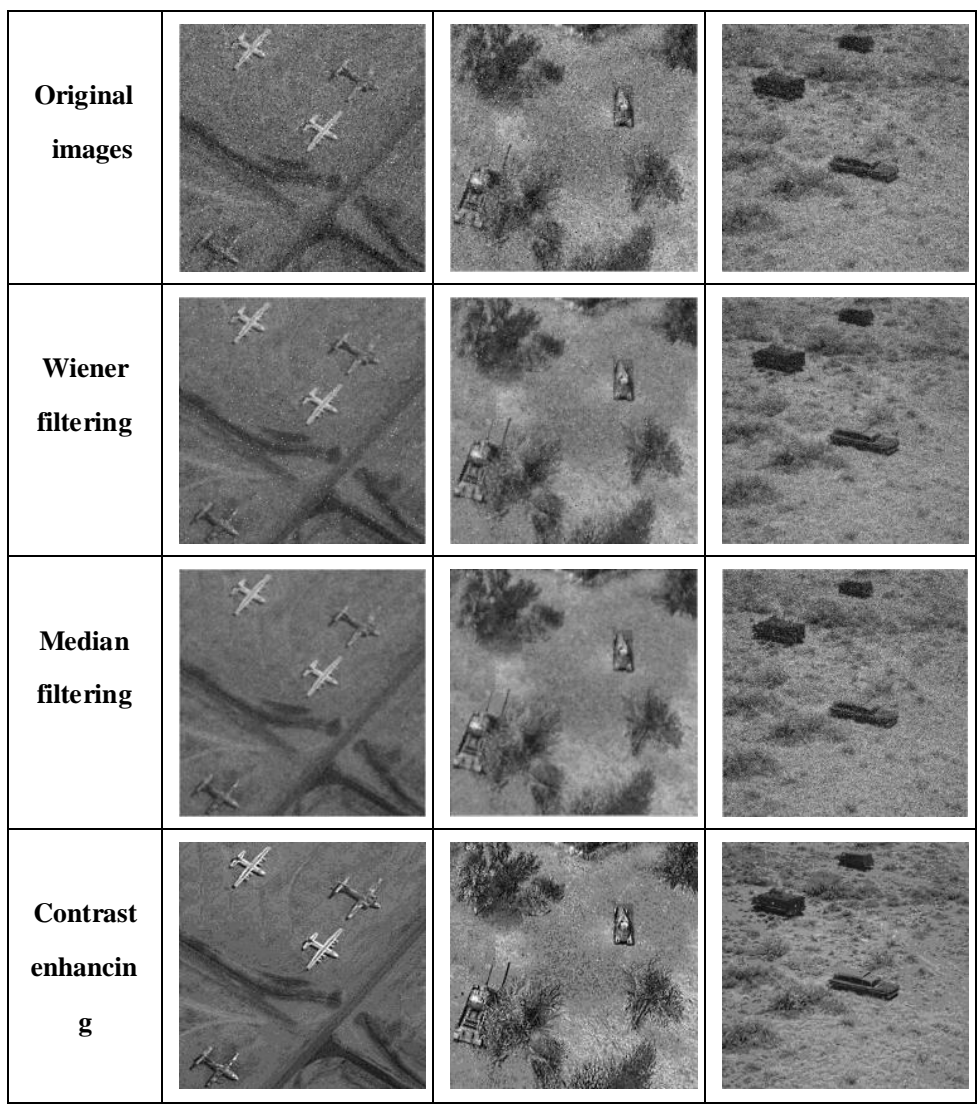


Our experiment is implemented on aerial images from USC-SIPI image database, and the software environment is Matlab7.8.0. $\mathrm{A}^{3 \times 3}$ window is applied when conducting image denoising while a disk-shaped structure element with 3 as its radius is used to enhance the contrast. Table I shows results of preprocessing.

In a $5 \times 5$ window, texture features are computed. Image segmentation is accomplished by FCM. Table II presents the results and comparison with paper [12].

That the number of clusters is 2 means an image is clustered into 2 portions, namely background and object areas.

Table III shows the results of man-made objects marking. Red areas contain detected objects. Some longer lines and sharper corners are preserved due to assumption 2. However, there's few distinction between our method and artificial marking. This may be caused by errors from extracting geometric structure features, or the following marking rules: in primary object location areas, choose a window containing both line edges and enough corners as object candidates, get a pixel by computing the average of coordinate of corners as the center of a $5 \times 5$ square to mark the objects.

Table IV shows the advantages and defects of proposed approach based on ATR evaluation index. The false alarm probability of proposed approach is usually high in respect that the chosen features of natural background are analogical with man-made objects. Consequently, this provides a guider for our further research.

\section{Conclusion}

This paper proposes an automatic man-made object detection approach, involving image denoising, contrast enhancing, feature extraction, and object marking. Man-made objects can be detected based on clustering texture feature and extracting geometric structure features. The experiments verify the rationality and validity of proposed approach.

From current researches, we may conduct further research on following facets: (1) Feature choosing and extracting. (2) Man-made object classification and recognition. Incorporating prior knowledge, objects can be tagged with semantic understanding and description, which pave the way for object recognition.

\section{Ackonwledge}

Thanks to USC-SIPI image database providing the test aerial images, and thanks to Professor M. J. Carlotto from Carnegie-Mellon University for his careful suggestion of our experiment. His comments and suggestion have contributed greatly to the depth and clarity of the presentation.

\section{References}

[1] Li Bo, Chen Qi-mei, Guo Fan. Freeway Auto-surveillance From Traffic Video [C]. 2006 6th International Conference on ITS Telecommunications Proceedings. 2006. pp: 167-170.

[2] Hinz S, Baumgartner A. Automatic extraction of urban road networks from multi-view aerial imagery [J]; ISPRS Journal of Photogrammetry \& Remote sensing, 2003, 58:83-98.

[3] BARNIV Y. Dynamic programming solution for detection dim moving target [J]. IEEE, Trans, AES 1994,30(1): 197-212.

[4] Jianxin Mei, Duan Shan, Qianqing Qin. Method for special targets detection based on support vector machines[J]. Geomatics and information science of Wuhan university 2004.29(10): 912-915.(in Chinese) 
[5] Caifei, Tudan. Survey on man-made object detection in visible imagery. Application Research of Computers, 2010, 27(7): 2430-2434. (in Chinese)

[6] E.Peli, “Contrast in complex images," J.Opt.Soc.Amer.A, 1990.vol.7,pp.2032-2040,

[7] Jinshan Tang, Scott Acton. Image Enhancement Using a Contrast Measure in the Compressed Domain [J]. IEEE signal processing LETTERS. 10(10). 2003.10. 289-292.

[8] Peter Howarth, Smfan M. Ruger. Evaluation of Texture Feature for Content-based Image Retrieval [C]. Third International Conference,CIVR2004: 326-334

[9] Smith S M. Brady M .Susan -A New Approach to Low Level Image Processing [J]. International Journal of Computer Vision 1997.23(1).

[10] Ridha Touzi, Arand Lopes, Pierre Bousquet. A Statistical and Geometrical Edge Detector for SAR Images [J]. IEEE Transaction on geoscience and remote sensing.1988. 11.26(6). 764-773.

[11] Yingying Chen, Zhaohui Yang, Qun Su. Automatic Recognition of Man-made Objects in SAR Images Using Support Vector Machines [J]. 2009 Urban Remote Sensing Joint Event 9(9): 78-83.

[12] M. J. Carlotto. A Cluster-based Approach for Detecting Manmade Objects and Changes in Imagery [J], IEEE Trans. Geoscience and Remote Sensing, 2005. 43(2):374-387

[13] E. Peli. “Contrast in complex images,” J. Opt. Soc. Amer. A. vol. 7, pp. 2032-2040, 1990.

TABLE II. RESULTS OF PRIMARY MAN-MADE OBJECTS LOCATION

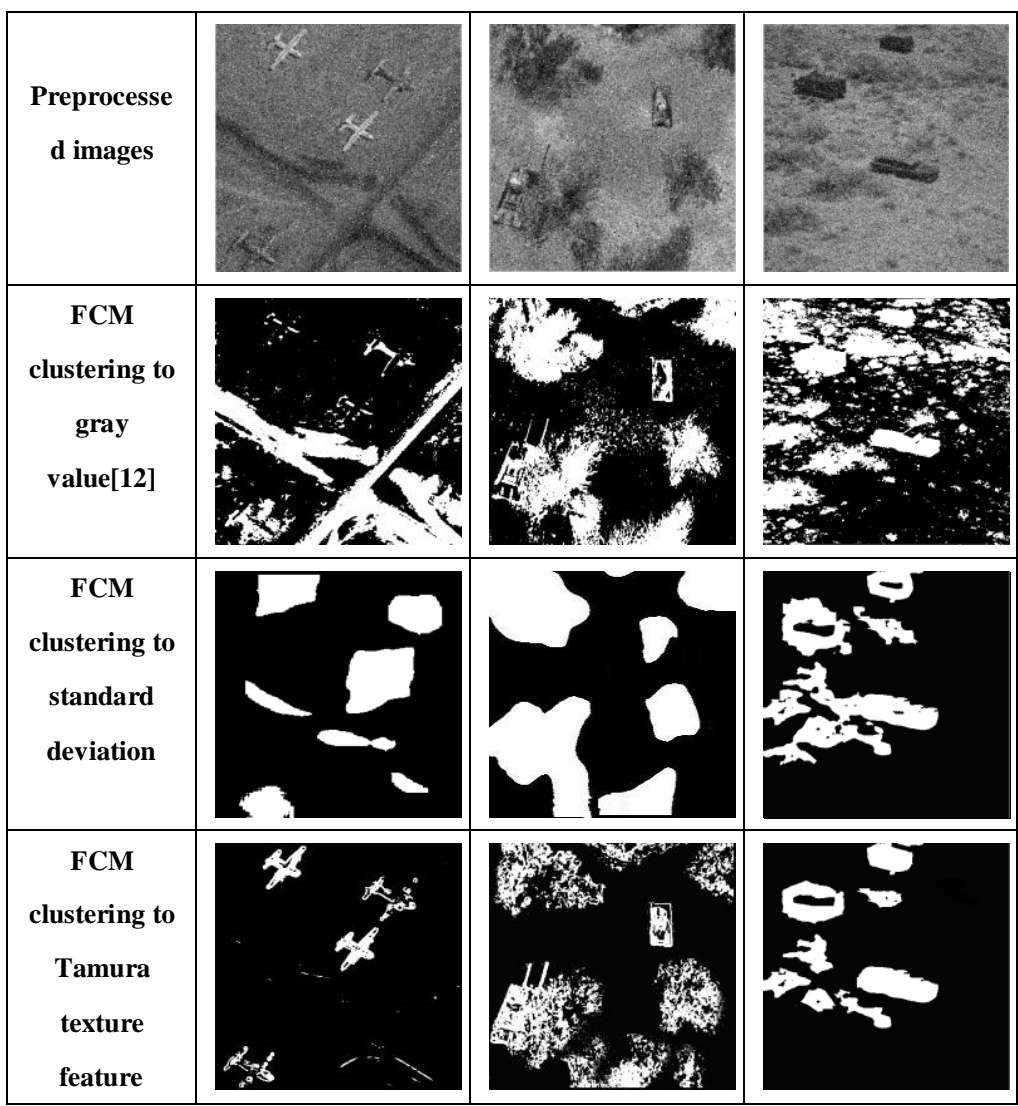


TABLE III. MAN-MADE OBJECTS MARKING (RED)

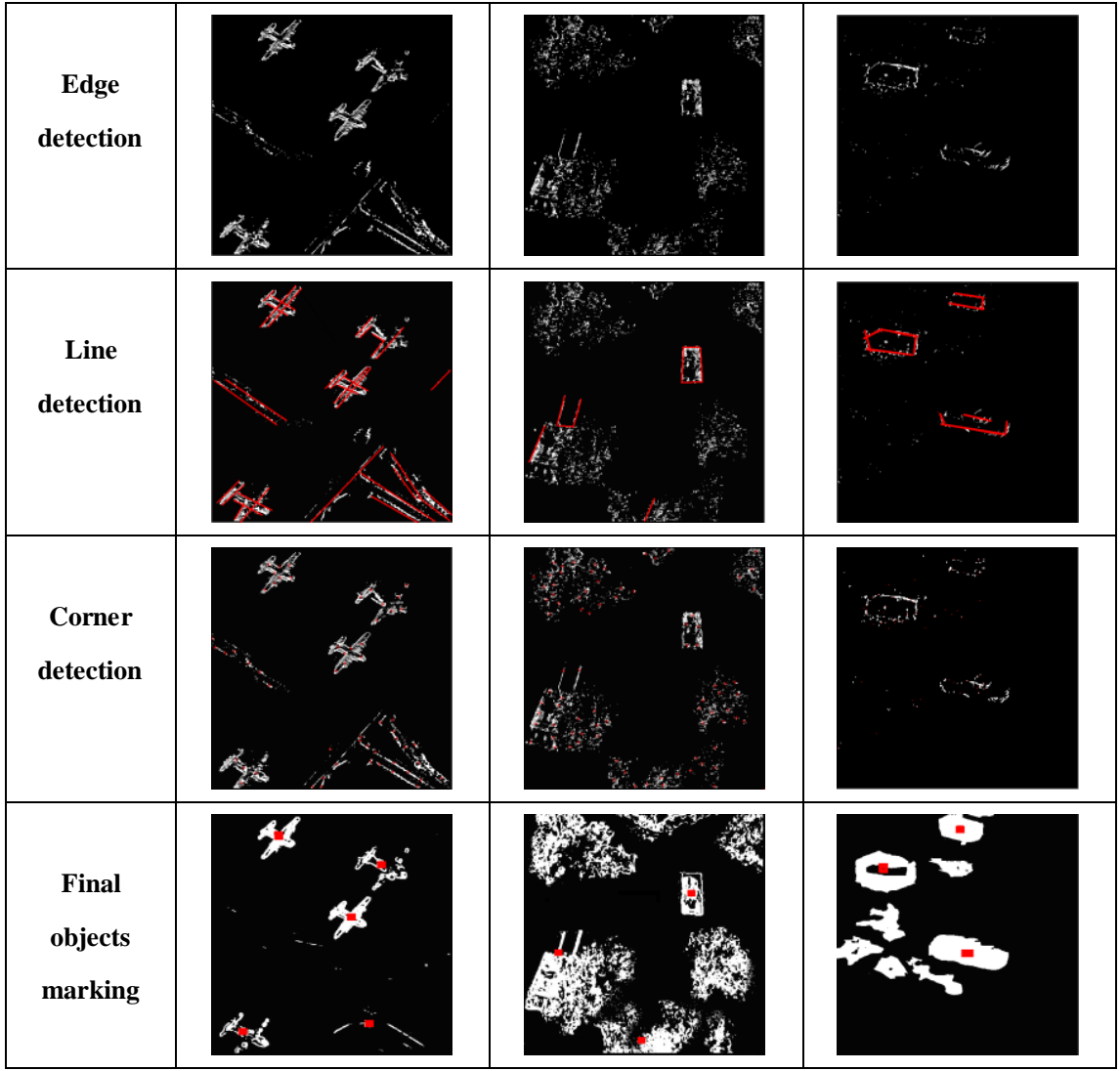


TABLE IV. EVALUATION OF PROPOSED METHOD

\begin{tabular}{|c|c|c|c|}
\hline \multicolumn{4}{|l|}{ Original images } \\
\hline \multicolumn{4}{|l|}{$\begin{array}{l}\text { Artificial man-made } \\
\text { objects marking }\end{array}$} \\
\hline Number of objects & 4 & 2 & 3 \\
\hline Automatic detected & 5 & 3 & 3 \\
\hline \multicolumn{4}{|l|}{ Automatic marking } \\
\hline Detection precision & excellent & general & excellent \\
\hline False alarm probability & $25 \%$ & $50 \%$ & 0 \\
\hline $\begin{array}{c}\text { Probability of missed } \\
\text { detection }\end{array}$ & 0 & 0 & 0 \\
\hline
\end{tabular}

